\title{
Design of Electronic Compass
}

\author{
Biqing $\mathrm{Li}^{1, \text { a }}$, Wenya Lai ${ }^{* 1,}$ a , Chongjun Yang ${ }^{1,}$, Shiyong Zheng ${ }^{2, b}$ \\ ${ }^{1}$ College of Mechanical and Electronic Engineering, Hezhou university, Hezhou Guangxi 542899, \\ China; \\ ${ }^{2}$ College of Computer Science and Information Engineering, Hezhou university, Hezhou Guangxi \\ 542899, China;
}

ajanliful@163.com, b229292710@qq.com

Corresponding Author: Wenya Lai

Keywords: electronic compass, sensor, microprocessor.

\begin{abstract}
The design is comprised of single-chip computer module, liquid crystal display module, sensor module, clock chip module and speech chip module. Magnetoresistive sensor is responsible for collection of magnetic field strength in specific direction, while clock chip is for calculation of data such as time and calendar. After that, the data are processed by microprocessor and then are displayed and uploaded by display screen. After debugging, accuracy rating of the compass could reach 3 degree, current azimuth will be displayed on LCD1602 liquid crystal display and will be broadcasted by speech chip. Comparing with traditional compass, this electronic compass is more portable and convenient with certain practical value.
\end{abstract}

\section{Introduction}

Current electronic science and technology has developed fast, so there are many choices on hardware designs of electronic compass, and each design has its own characteristics. So we can choose corresponding hardware circuit according to actual situation.

In the three-dimensional display of a regional earth magnetic field, $\mathrm{x}$-axis and $\mathrm{y}$-axis parallel to the floor, while direction $\mathrm{X}$ and $\mathrm{Y}$ is vertical to horizontal plane $\mathrm{X}$, which means geomagnetic field intensity. Direction of azimuthal angel is intersection angel between dip angel between magnetic pole and magnetic declination. The value of intersection angel between the Antarctic Pole and $\mathrm{x}$-axis is $(a+\lambda)$, so if we know the value of azimuthal angel $a$, we could figure out the direction of horizontal plane. From tan $a=$ Hey/Hex, if we want to know the tangent value, we must calculate the ratio of Hey and Hex. In this way, we could check and correct the result that the intersection angel between he and horizontal plane is horizontal inclination $\delta$. Magnetic field sensor could not only measures the value of Hex, Hey and Hez, it could also convert different value to different electric signal.

Measured signal will be conveyed to LCD1602 liquid crystal display to display after processed by single-chip microcomputer, at the same time, speech module will be responsible for broadcast of current azimuthal angel.

Combined with design choice, we could get the overall scheme design diagram. Details are as below:

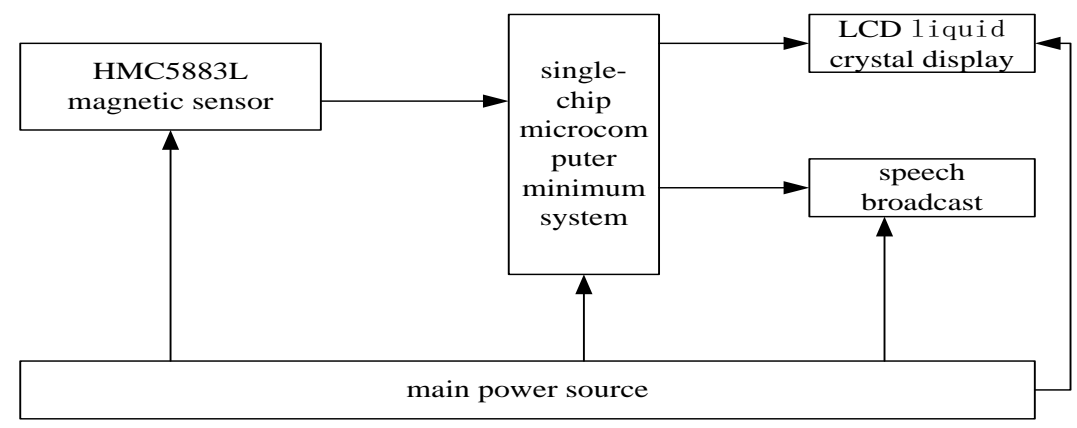

Graph 1 Overall scheme design diagram 


\section{Software Design}

Communication between HMC5883L and single-chip microcomputer

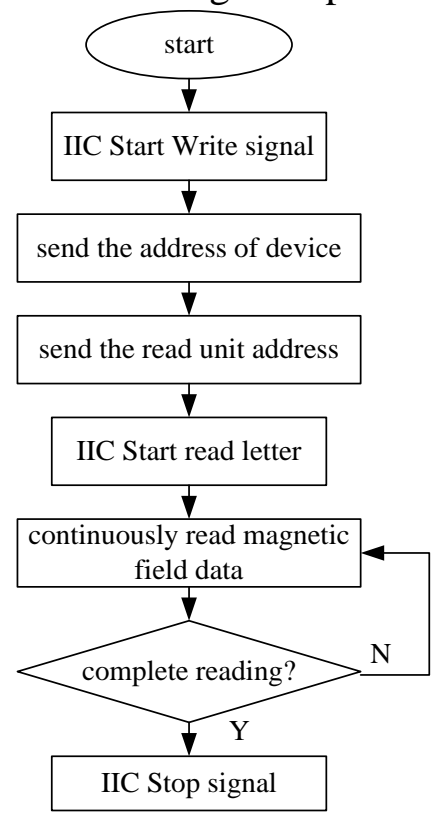

Graph 2 HMC5883L module and microprocessor communication diagram

HMC5883L is an integrated digital sensor of ICC bus interface. However interface as STC89C52 single-chip microcomputer will choose to communicate with ICC and with HMC58831 microprocessor regularly, but HMC58831 could choose to read in single byte and multibyte. In this design, it will read in multibyte and it the values of XYZ will be read into single-chip microcomputer in a time. Detail communication diagram is as below:

\subsection{LCD1602 human-computer interface}

Display information of human-computer interface includes information of intersection angel (degree measure) between current location and south geographical pole, calendar, time and etc.. It will also calculate intersection angel (radian) between the magnetic field intensity component of magnetic location in $\mathrm{x}$-axis and $\mathrm{y}$-axis and north geographical pole. The location will be distinguished based on angel limit set before after got angel degree, and the location will be displayed on LCD1602.

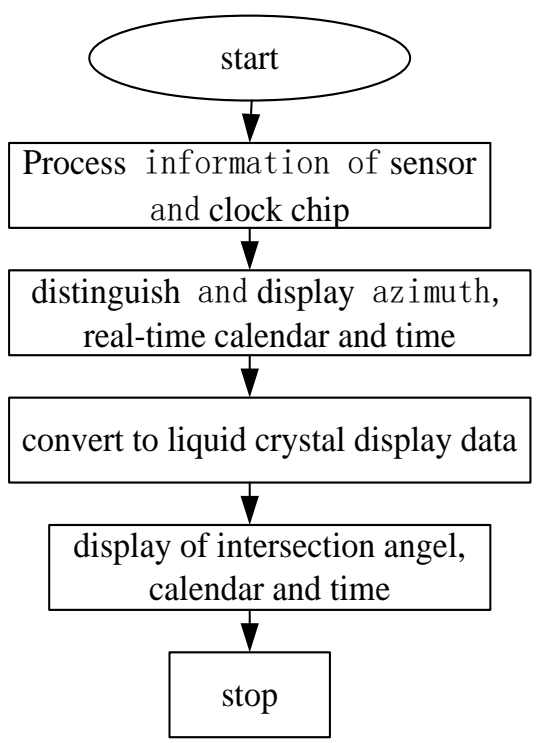

2.2 System overall process

Graph 3 LCD1602 human-computer interface diagram

Information acquired by HMC5883L will be processed by microprocessor, then convert to liquid crystal display data that will be displayed on LCD1602, at last get the azimuth and angel information. At the same time, real-time time and date will be displayed on LCD1602 liquid crystal 
display at the same time through DS1302. And the speech chip will collect speech signal, current azimuth will be broadcasted through horn after process. The diagram is as below:

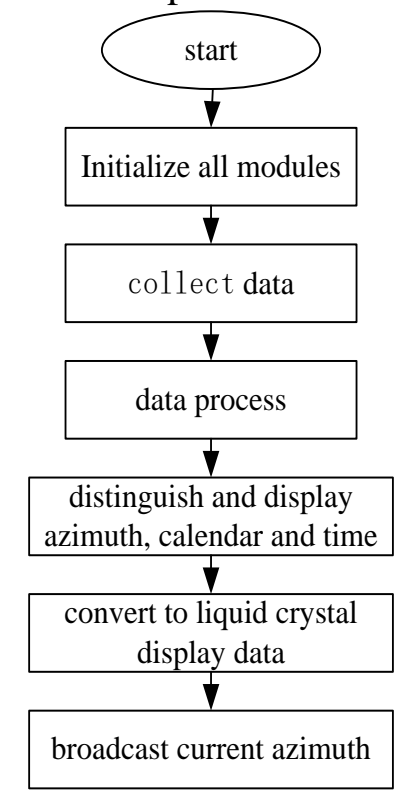

Graph 4 System overall process diagram

\section{Conclusion}

The compass module designed in this article, simple in design and with good integration and measuring error within 3 degrees, could be used for direction judgment of navigation and mountainous exploration. Low power consumption microprocessor in the compass module improves the ability of the device. But design of HMC5883 sensor just includes value X and Y, which could only be accurately measured when the system is in horizon level. If HMC5883L could be combined with z-axis in a three dimensional direction, current azimuth could be accurately measured in any pose.

This work is supported the following fund :

2016 The project of improving the basic ability of young teachers in Colleges and universities in Guangxi:"Design and development of electronic commerce platform of agricultural products based on Semantic Technology”(No,KY2016YB455).

2016 Guangxi higher education teaching reform project: "Exploration on the cultivation mechanism of the teaching characteristics of Communication Engineering Specialty Based on ZTE ICT education platform"

2015 college students' innovative training program: "Research on the application of value added travel experience in the mobile terminal of the 'ethnic custom travel' in Guangxi" (No 201511838070);\&\& “The design and development HeYuanTong Campus Mobile Phone APP based on Android” (No 201511838034).

2015 Teaching case project construction project of hezhou university: "Tourist positioning and guiding system project of Huangyao scenic";\&\& "Development and application of the information management system of 'Huang Yao Tong'”.

Project of scientific research and technology development project of Hezhou: "Design and implementation of agricultural products e-commerce platform based on Semantic Technology" (No,Hekeneng 1506006)

Scientific research project of hezhou university:"Research and development of E-government platform based on mobile terminal” (No 2015ZZZK03);

Reform in Education project of hezhou university: "Internet of things"(No hzxytszy201501); "Research on application personnel training model of Communications for SMEs"(No hzxyjg201525);

Master degree discipline construction scientific research and Cultivation Project: "Research on the social work service of the teenagers' Network Addiction”(No 2015SHGZ005) \&\&"Research on 
the intelligent development of rural tourism in Guangxi based on the Internet of things" (No 2015MTA16);

\section{References}

[1]. Hu Ningbo, Li Jian, Zhao Juyun, Electronic Compass Design Based on HMC5883L. Sensor World.11 (635-38) 2011.

[2]. Guo Jianrui, Electronic Compass Design Based on Magnetic Resistance Chip and MPS430 Single-chip Microcomputer [J].Information and Electronic Engineering.81 (12-14） 2010.

[3]. Shao Tingting, Ma Jiancang. Study on Compensate Algorithm of Compass Inclination and Deviation of Electronic Compass [J].Journal of Sensor Technology, 2007 